\title{
6 Distributed space-time block codes
}

Matthew C. Valenti and Daryl Reynolds

\section{$6.1 \quad$ Introduction}

In this chapter, we consider space-time coding strategies for multiple-relay cooperative systems that effectively harness available spatial diversity. More specifically, the goal is to examine ways to efficiently forward signals from multiple relays to the destination while addressing the important practical issue of synchronization among the relays. We assume a general two-phase transmission protocol as illustrated in Figure 6.1. In the first phase of the protocol, the source broadcasts a message which is received by the relays and (possibly) the destination. During the second transmission phase, a subset of the relays, possibly in conjunction with the source, transmit additional information to the destination. This protocol is useful in practical scenarios where signals received at the destination due to transmissions directly from the source (Phase 1) will not carry enough useful information because of noise, fading, and/or interference. It is expected that Phase 2 will dramatically increase reliability of the system, but

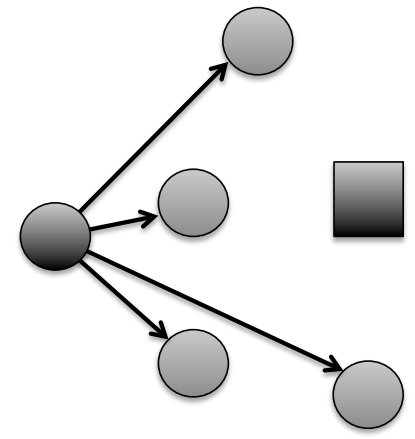

Phase I

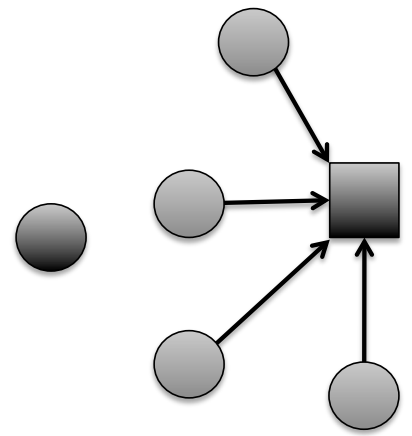

Phase II

Figure 6.1: Illustration of the two-phase transmission protocol using a distributed space-time code. In the first phase (left subfigure) the source transmits to several relays, while in the second phase (right subfigure), the relays simultaneously transmit to the destination. 
if the symbols cannot be decoded correctly after the second phase, the protocol can re-start by returning to Phase 1 or Phase 2 .

The primary problem associated with forwarding information from multiple relays to the destination is determining how the information should be spread out among the relays over space and time. This is analogous to the classic spacetime coding problem in point-to-point multiple transmit antenna systems, and so it is often called the distributed space-time coding problem. Similar to the point-to-point scenario, performance can vary dramatically based on the coding scheme, so care must be taken to achieve high performance in terms of diversity and coding gain while accounting for the practical limitations of cooperative systems. In fact, poorly-designed distributed space-time coding schemes may perform worse than point-to-point systems, especially considering that the twostage protocol consumes additional degrees of freedom (time resources) relative to direct transmission.

A conservative solution to the distributed space-time coding problem is for each terminal that participates in the second phase to transmit using orthogonal subchannels, which could be implemented using disjoint time slots, different frequency subbands, or orthogonal spreading codes (assuming that they can be synchronized). When disjoint time slots are used, this strategy is a distributed form of delay diversity [22]. However, there are two problems associated with this approach: 1) it is known that orthogonalizing system resources is suboptimal [18], and 2) enforcing orthogonalization in time, frequency, or code space would require significant additional signaling overhead, e.g., feedback and/or synchronization, and, in many cases, may not even be possible.

A more efficient, albeit more aggressive, approach is for the terminals transmitting in the second phase to use a distributed space-time block code ${ }^{1}[10,15]$. There are two main varieties of forwarding mechanisms that can be used with distributed space-time coded systems: decode and forward (DF) [10] and amplify and forward (AF) [8]. In DF protocols, the source's signal is encoded with a forward error correcting code that must be successfully decoded by a relay before that relay may participate in the transmission of the distributed space-time code. After decoding, the information is re-encoded and re-modulated. A linear combination of the re-modulated symbols and their conjugates are transmitted by each participating relay. While the DF protocol avoids error propagation or the amplification of noise received over the source-relay channel, its implementation is complicated by fact that the set of relays that are able to decode and so participate in the space-time code in the second phase is random and unknown to the system. Therefore, unlike point-to-point multiple antenna systems, extra care must be taken to assure that the relays coordinate in the transmission of the space-time codeword.

${ }^{1}$ Distributed space-time trellis codes can be used as well, but we focus here on block codes and refer the interested reader to, for example, [13]. 
With AF protocols, a fixed number of relays participate in the second phase by transmitting a linear combination of the time samples of the noisy received signal (not decoded symbols) they receive from the source. Because the number of relays is fixed, AF does not have to manage which relays transmit which part of the space-time codeword, unlike DF. However, with AF the noise received over the source-relay channels is amplified, which has the potential to degrade performance.

There are a number of challenges associated with practical distributed spacetime code implementation, some of which are shared with conventional spacetime coding used in point-to-point multiple antenna systems, but some are unique to the distributed scenario. As with conventional space-time codes, distributed codes typically (but not always) require the destination receiver to have knowledge of the channels between the relays and the destination. Unlike conventional space-time codes, however, distributed space-time codes are subject to some challenging synchronization issues. Because the transmitters are widely separated and have different time references, and due to differences in the propagation delay between the relays and the destination, the different signals received by the destination will generally be offset in time. Although this problem can perhaps be overcome with appropriate synchronization protocols, it can more effectively be handled with delay diversity, delay-tolerant distributed space-time codes, or space-time spreading.

The remainder of this chapter is as follows. In Section 6.2, we give a system model that will be used through the chapter. In Section 6.3, we review concepts related to fixed space-time block codes. In Section 6.4, we describe the DF protocol, while in Section 6.5 we describe the AF protocol. We discuss synchronization issues in Section 6.6, and conclude in Section 6.7.

\subsection{System model}

Consider a wireless network consisting of a source node, a destination node, and a set of $R$ relay nodes $\mathcal{N}=\left\{N_{i}: 1 \leq i \leq R\right\}$. Each node has a half-duplex radio and a single antenna. Messages are transmitted according to a two-phase protocol. During the first phase, a signal of duration $T_{1}$ symbol periods is broadcast by the source and received by the relays. During the second phase, a subset of the relays will simultaneously, but perhaps not synchronously, transmit signals of duration $T_{2}$ symbol periods, and the destination will receive a noisy sum of the relay signals. After approximately $T=T_{1}+T_{2}$ consecutive symbol periods (depending upon transmission and channel delays), the source will move on to the next message (or a retransmission of a failed message). For ease of exposition, we assume that there is no direct link between the source and destination, although the protocols and performance analyses can easily be generalized to allow for such a link. 
We adopt a discrete-time model, whereby the signal transmitted by the source during the first phase is represented by the vector $\mathbf{s}=\left[s_{1}, \ldots, s_{T_{1}}\right]^{t}$. The individual symbols $s_{\ell}, 1 \leq \ell \leq T_{1}$ are each drawn from a complex constellation $\mathcal{X}$ of $M$ symbols. The signal constellation is normalized so that its average energy is unity, i.e., $\frac{1}{M} \sum_{s \in \mathcal{X}}|s|^{2}=1$. The normalized signal is amplified and transmitted by the source with power $P_{1}$ during the first phase. Let $f_{i}$ represent the complex gain of the channel between the source and node $N_{i}$. Then the signal received by node $N_{i}$ during the first phase is

$$
\mathbf{r}_{i}=f_{i} \sqrt{P_{1}} \mathbf{s}+\mathbf{v}_{i}
$$

where $\mathbf{v}_{i}=\left[v_{i, 1}, \ldots, v_{i, T_{1}}\right]^{t}$ is a noise vector containing independent circularlysymmetric complex Gaussian random variables with zero mean and unit variance.

In the second phase, a subset $\mathcal{K} \subseteq \mathcal{N}$ of the relays will simultaneously, but perhaps without symbol-level synchronization, transmit to the destination. During this phase, node $N_{i} \in \mathcal{K}$ will transmit a signal represented by the discrete-time vector $\mathbf{t}_{i}=\left[t_{i, 1}, \ldots, t_{i, T_{2}}\right]^{t}$ with power $P_{2}$. When the signals are perfectly synchronized, the signal received at the destination will be

$$
\mathbf{x}=\sum_{i: N_{i} \in \mathcal{K}} g_{i} \sqrt{P_{2}} \mathbf{t}_{i}+\mathbf{w}
$$

where $g_{i}$ is the complex gain of the channel between node $N_{i}$ and the destination, and the noise vector $\mathbf{w}=\left[w_{1}, \ldots, w_{T_{2}}\right]^{t}$ contains independent circularlysymmetric complex Gaussian random variables with zero mean and unit variance. When the signals are not synchronized, the model must be generalized to account for time offsets.

In general, the power $E\left[\left|f_{i}\right|^{2}\right]$ and $E\left[\left|g_{i}\right|^{2}\right]$ of the channel gains $f_{i}$ and $g_{i}$ will depend on the topology of the network and the propagation characteristics of the wireless channel, and will usually be unequal. However, for ease of exposition, we make the simplifying assumption that the $f_{i}$ 's and $g_{i}$ 's are independent and identically distributed (i.i.d.). In particular, each $f_{i}$ and $g_{i}$ is assumed to be a circularly-symmetric complex Gaussian with zero mean and unit variance, so that their envelopes $\left|f_{i}\right|$ and $\left|g_{i}\right|$ are Rayleigh distributed. The coefficients $f_{i}$ are held fixed for the transmission of the signal $\mathbf{s}$, and the coefficients $g_{i}$ are held constant for the transmission of the $\mathbf{t}_{i}$, i.e., we assume a Rayleigh block fading model.

Just as the channel gains might have unequal powers, the powers $P_{2}$ transmitted by the relays can, in general, be selected such that they are unequal, in which case our notation would need to be modified to indicate the different powers. However, in the following discussion we impose the simplifying limitation that all relays transmit with the same power $P_{2}$, which is optimal when the average channel powers are all equal and the transmitters operate without channel state information.

Note that this is a fairly general model which leaves unaddressed several critical design and implementation issues. For example, the composition of the set $\mathcal{K}$ will 
depend on the protocol being used. In DF protocols, $\mathcal{K}$ contain only relays that successfully decoded the source's transmission, while in AF protocols, $\mathcal{K}$ may contain any (or all) of the relays. Another key issue is the selection of the signals $\mathbf{t}_{i}$ that are to be transmitted by the nodes in $\mathcal{K}$ during the second phase of the protocol. These signals can be jointly coded, but in a distributed way, using a space-time code, or they can use simpler strategies, e.g., delay diversity or spacetime spreading. We will discuss all of these options in this chapter. Finally, the allocation of power between $P_{1}$ and $P_{2}$ for the two transmission phases and the allocation of time over the two time phases are protocol-dependent optimization problems that must be solved to maximize performance.

\subsection{Space-time block codes}

As described in the Introduction, one of the primary problems associated with forwarding information from relays to a destination in a cooperative wireless network is how information is transmitted from the relays over time, i.e., the space-time transmission scheme. One natural strategy is to extend the concept of space-time block codes, typically used for point-to-point multiple transmit antenna systems, to relay networks, where they are called distributed space-time block codes. We begin with a description of conventional space-time block codes under the quite general linear dispersion paradigm [6].

Suppose for the moment that the first-phase transmission is perfectly received by all $R$ relays. Under the linear dispersion paradigm, the $i^{\text {th }}$ relay transmits a linear combination of the $T_{1}$ symbols in $\mathbf{s}$ and their complex conjugates,

$$
\mathbf{t}_{i}=A_{i} \mathbf{s}+B_{i} \overline{\mathbf{s}}
$$

where $\overline{\mathbf{s}}$ is the column vector containing the complex conjugates of $\mathbf{s}$ and the complex $T_{2} \times T_{1}$ matrices $A_{i}$ and $B_{i}$ are called dispersion matrices. These matrices define the space-time code. Each nonzero matrix $A_{i}$ or $B_{i}$ is constrained to be unitary, and since the signal set $\mathcal{X}$ is normalized to unit energy, the symbols transmitted by the relays will also have unit energy.

The family of space-time block codes that can be represented by (6.3) are called linear dispersion (LD) codes [6]. This family of codes includes many wellknown space-time codes as special cases. For example, one linear dispersion code that has been proposed for cooperative communications with $R=2$ relays is described by the dispersion matrices [9]

$$
A_{1}=\left[\begin{array}{cc}
+1 & 0 \\
0 & +1
\end{array}\right], A_{2}=0_{2 \times 2}, B_{1}=0_{2 \times 2}, B_{2}=\left[\begin{array}{cc}
0 & -1 \\
+1 & 0
\end{array}\right]
$$

where $0_{m \times n}$ is a $m \times n$ matrix of all-zeros. This code is simply a transpose of the well-known Alamouti space-time block code [1].

For many codes of interest, including the one specified by (6.4), either $A_{i}$ or $B_{i}$ is a matrix of zeros for every $i$. This means that a particular relay will transmit 
a linear combination of the symbols in $\mathbf{s}$ or $\overline{\mathbf{s}}$, but not both. If we define

$$
C_{i}= \begin{cases}A_{i}, & \text { if } B_{i}=0 \\ B_{i}, & \text { if } A_{i}=0\end{cases}
$$

and

$$
\mathbf{s}^{(i)}= \begin{cases}\mathbf{s}, & \text { if } B_{i}=0 \\ \overline{\mathbf{s}}, & \text { if } A_{i}=0\end{cases}
$$

then we can write (6.3) more compactly as

$$
\mathbf{t}_{i}=C_{i} \mathbf{s}^{(i)} .
$$

Assume that nodes $N_{1}, \ldots, N_{R}$ participate in the second phase transmission with the same power $P_{2}$. The signal received at the destination will be

$$
\mathbf{x}=\sqrt{P_{2}} S \mathbf{h}+\mathbf{w}
$$

where

$$
S=\left[\begin{array}{lll}
C_{1} \mathbf{s}^{(1)} & \ldots & C_{R} \mathbf{s}^{(R)}
\end{array}\right]
$$

is the $T_{2} \times R$ space-time codeword, and

$$
\mathbf{h}=\left[\begin{array}{lll}
g_{1} & \ldots & g_{R}
\end{array}\right]^{t}
$$

is the channel vector. The maximum-likelihood (ML) detector at the destination estimates the source signal as

$$
\hat{\mathbf{s}}=\arg \min _{\mathbf{s} \in \mathcal{X}^{T_{1}}}\left\|\mathbf{x}-\sqrt{P_{2}} S \mathbf{h}\right\|
$$

where $\|\cdot\|$ indicates the Frobenius norm.

Unless the distributed space-time code, described by the set of all possible codewords $S$, has some special structure, ML detection will have exponential complexity in the number of source symbols $T_{1}$. Fortunately, several classes of codes allow reduced complexity for ML decoding, including the well-known orthogonal design family [15], whose orthogonal structure allows for decoupling of the symbols in the codeword, permitting for symbol-by-symbol ML detection with linear complexity in $T_{1}$. The (Alamouti) code given by (6.4) is one example of an orthogonal design. Another orthogonal design which has been applied to cooperative diversity with $R=4$ relays is described by the dispersion matrices 
$[9]$

$$
\begin{array}{ccc}
A_{1}=\left[\begin{array}{lll}
1 & 0 & 0 \\
0 & 0 & 0 \\
0 & 0 & 0 \\
0 & 0 & 0
\end{array}\right], & A_{2}=\left[\begin{array}{lll}
0 & 1 & 0 \\
0 & 0 & 0 \\
0 & 0 & 0 \\
0 & 0 & 0
\end{array}\right], A_{3}=\left[\begin{array}{lll}
0 & 0 & 1 \\
0 & 0 & 0 \\
0 & 0 & 0 \\
0 & 0 & 0
\end{array}\right] \\
A_{4}=\left[\begin{array}{ccc}
0 & 0 & 0 \\
0 & 0 & 1 \\
0 & 1 & 0 \\
-1 & 0 & 0
\end{array}\right], B_{1}=\left[\begin{array}{ccc}
0 & 0 & 0 \\
0 & -1 & 0 \\
0 & 0 & 1 \\
0 & 0 & 0
\end{array}\right], B_{2}=\left[\begin{array}{lll}
0 & 0 & 0 \\
0 & 0 & 0 \\
0 & 0 & 1
\end{array}\right] \\
B_{3}=\left[\begin{array}{ccc}
0 & 0 & 0 \\
0 & 0 & 0 \\
-1 & 0 & 0 \\
0 & -1 & 0
\end{array}\right], B_{4}=0_{4 \times 3} .
\end{array}
$$

Note that for this code, only $B_{4}$ is all-zeros and thus the model given by (6.5) through (6.10) must be generalized slightly. See $[8,9]$ for details.

The rate of a space-time block code is $T_{1} / T_{2}$, and a code is said to be full rate if it has a rate of unity. While the rate of the code specified by (6.4) is unity, the rate of the $R=4$ code specified by (6.12) is only $3 / 4$. No full-rate orthogonal STBC exists for $R>2$ when complex symbols are used [11], although reducedrate orthogonal codes can be designed for any number of transmit antennas. Thus, for $R>2$, the convenience (linear ML decoding complexity) of using an orthogonal design comes at the cost of reduced spectral efficiency. An alternative to using orthogonal designs is to use quasi-orthogonal designs [7], which can achieve full rate with four antennas with higher complexity than orthogonal designs, but much lower than worst-case ML complexity. The additional complexity over orthogonal designs is because quasi-orthogonal codes reduce the ML detection problem to the joint detection of pairs of complex symbols, whereas orthogonal designs reduce it to the detection of individual complex symbols. The ML detector for a quasi-orthogonal code thus requires that each of the $T_{1} / 2$ pairs of symbols be compared against $M^{2}$ hypothesis. An example quasi-orthogonal 
STBC considered for cooperative-diversity with $R=4$ relays is given by [9]

$$
\begin{array}{ccc}
A_{1}=\left[\begin{array}{llll}
1 & 0 & 0 & 0 \\
0 & 1 & 0 & 0 \\
0 & 0 & 1 & 0 \\
0 & 0 & 0 & 1
\end{array}\right], & A_{2}=0_{4 \times 4}, \quad A_{3}=0_{4 \times 4} \\
A_{4}=\left[\begin{array}{cccc}
0 & 0 & 0 & 1 \\
0 & 0 & -1 & 0 \\
0 & -1 & 0 & 0 \\
1 & 0 & 0 & 0
\end{array}\right], \quad B_{1}=0_{4 \times 4}, \quad B_{2}=\left[\begin{array}{cccc}
0 & -1 & 0 & 0 \\
1 & 0 & 0 & 0 \\
0 & 0 & 0 & -1 \\
0 & 0 & 1 & 0
\end{array}\right] \\
B_{3}=\left[\begin{array}{cccc}
0 & 0 & -1 & 0 \\
0 & 0 & 0 & -1 \\
1 & 0 & 0 & 0 \\
0 & 1 & 0 & 0
\end{array}\right], \quad B_{4}=0_{4 \times 4} .
\end{array}
$$

In Figure 6.2, four systems are compared by plotting the bit error rate (BER) of each system as a function of the signal-to-noise ratio (SNR). Three values of $R$ are considered, $R=\{1,2,4\}$, and all systems transmit with a spectral efficiency of 3 bits-per-second-per-Hertz (bps/Hz). The signals are transmitted over Rayleigh fading channels, and the power is split evenly across the $R$ transmit antennas. Since the noise power is unity, the transmitted power is $P_{2}=S N R / R$. The $R=1$ system represents conventional point-to-point communications between a pair of terminals, each with a single antenna and no space-time coding. The $R=2$ system uses the transposed Alamouti code given by (6.4). Two systems are compared for use with $R=4$ transmitting antennas, the orthogonal code of (6.12) and the quasi-orthogonal code (6.13). The rate of the orthogonal space time code used with $R=4$ antennas is $3 / 4$, while the rates of the other STBC are all unity. In order for the spectral efficiency to be maintained at $3 \mathrm{bps} / \mathrm{Hz}$, gray-labeled 8-PSK modulation is used for the full rate systems (including the system with no space-time coding), while gray-labeled 16-QAM modulation is used for the rate $3 / 4$ system. The worst-performing system is the one that uses just one transmit antenna $(\mathrm{R}=1)$, while the next worst-performing system is the one with two transmit antennas $(\mathrm{R}=2)$. The two systems four transmit antennas $(\mathrm{R}=4)$ exhibit the best performance.

The most significant feature to notice in Figure 6.2 is the steepness of the curves. At high SNR, these curves become straight lines, implying that asymptotically there is a a linear relationship between the logarithm of the error probability and the SNR expressed in $\mathrm{dB}$. The negative slope is called the diversity of the system, also called the diversity order or diversity gain. Inspection of the diagram reveals that the diversity of the $R=1$ system is equal to one (i.e., the BER drops by an order of magnitude with every decade of SNR), while the diversity of the $R=2$ system is equal to two. Although the SNR is not sufficiently high in the figure to show it, the diversity of the two $R=4$ systems are both equal 


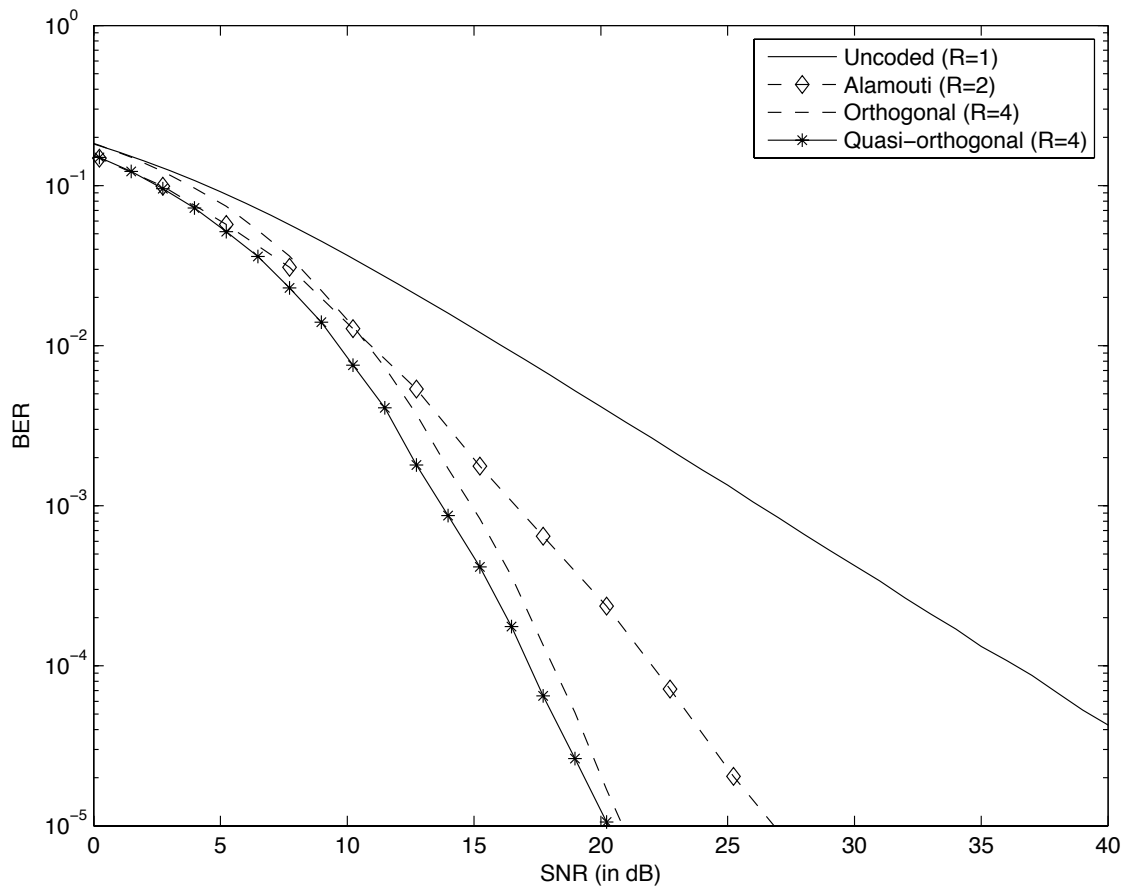

Figure 6.2: Bit error rate (BER) performance of four systems: An uncoded system with $R=1$ transmit antenna, an Alamouti-coded system with $R=2$ transmit antennas, an orthogonal STBC with $R=4$ transmit antennas, and a quasi-orthogonal STBC with $R=4$ transmit antennas. In each case, the spectral efficiency is $3 \mathrm{bps} / \mathrm{Hz}$ and the signals are transmitted over independent Rayleigh fading channels.

to four. A system with $R$ antennas is said to have full diversity if its diversity order is $R$. Thus, all four systems shown in Figure 6.2 exhibit full diversity, as seen by the fact that their error probabilities decay proportional to $1 / S N R^{R}$.

The performance of a space-time coded system can be determined by analyzing the pairwise error probability (PEP) between all pairs of distinct space-time codewords $S_{k}$ and $S_{\ell}$. The PEP can be bounded by, for example, a Chernoff bound. By taking the limit with respect to the SNR, the diversity order is then determined. Full diversity is achieved by assuring that $S_{k}-S_{\ell}$ is full rank for all $k \neq \ell[15]$.

Linear dispersion codes need not be orthogonal or quasi-orthogonal. For instance, the linear dispersion codes presented in [6] were designed to maximize the mutual information between the transmitter and receiver under a power constraint. However, such codes do not lend themselves to the very simple decoder structures that are possible with orthogonal or quasi-orthogonal codes. While a brute-force ML decoder requires comparison against all $M^{T_{1}}$ hypothesis, the complexity can be greatly reduced by using a sphere decoder [2]. Another option 
is to use the designs in [17], which permit decoupled symbol-by-symbol decoding and full-rate at the cost of reduced diversity.

\subsection{Decode-and-forward distributed STBC}

Returning to the two-phase relay network configuration, consider the case that the first-phase transmission must take place over a channel that is corrupted by noise and fading. Now, there is no guarantee that any particular node will receive the transmission correctly. With a decode-and-forward protocol, the source will encode its transmissions with a channel code. Each relay will attempt to decode using the signal it receives, and can only participate in the second-phase transmission if it successfully decoded the message sent by the source. The condition that a node can only transmit in the second phase if it successfully decodes the message requires that the code be used not only as an error correcting code, but also as an error detecting code (i.e., the relay needs to detect the existence of uncorrectable errors). The set of nodes $\mathcal{K}$ that successfully decoded the source's message and may transmit during the second phase is called the decoding set [10], and the number of nodes in the decoding set is $K=|\mathcal{K}|$.

During the second phase, nodes in $\mathcal{K}$ transmit using a distributed space-time code. A major complicating factor is that the size of the decoding set is random, yet the space-time code must be designed with a certain number of transmitting antennas in mind. Because of this, the number of relays that actually transmit should be limited to the maximum number of antennas supported by the spacetime code, which we denote $K_{\max }$. It is possible that $K<K_{\max }$, which means that there will not be enough relays participating in the second phase to use the entire space-time code. This implies that the space-time code should be "scale free", meaning that it still offers the maximum possible diversity even if some of the transmitting antennas are not used. When $K<K_{\max }$, the maximum possible diversity order is reduced from $K_{\max }$ to $K$. It is known that orthogonal space-time codes have this scale-free property [9].

If relay $N_{i} \in \mathcal{K}$ transmits during the second phase, then it does so by transmitting a signal vector $\mathbf{t}_{i}$ of the form given by (6.3), where $\mathbf{s}$ is the signal obtained by decoding, re-encoding, and re-modulating, and the $A_{i}$ and $B_{i}$ are the dispersion matrices currently assigned to that relay. Note that since the composition of the set of transmitting nodes changes after each source transmission, the set of dispersion matrices assigned to a particular relay may also change. The protocol must be careful to make sure that each transmitting relay is allocated a distinct set of dispersion matrices. When either $A_{i}$ or $B_{i}$ is all zeros for all $i$, the received signal at the destination is as given by (6.8), where the columns of the space-time codeword $S$ will be the signals transmitted by the relays. When $K<K_{\max }$, fewer relays transmit than there are columns in $S$ and $K_{\max }-K$ columns in $S$ will be all-zeros. 


\subsubsection{Performance analysis}

The performance of a DF system depends on the error control code or codes used. If the system uses a capacity-approaching code, such as a turbo code or a lowdensity parity-check (LDPC) code, then the codeword error rate over a particular link may be approximated by the information-outage probability of that link. The information-outage probability is the probability that the conditional mutual information between the channel input and output is below some threshold. For the first phase, the channel between the source and each relay is an additive white Gaussian noise (AWGN) channel when it is conditioned on the fading gain $f_{i}$. The conditional mutual information between the signal transmitted by the source and the signal received by the $i^{t h}$ relay is given by

$$
I\left(\mathbf{s}, \mathbf{r}_{i} \mid f_{i}\right)=\log _{2}\left(1+P_{1}\left|f_{i}\right|^{2}\right)
$$

where $P_{1}\left|f_{i}\right|^{2}$ is the "instantaneous" SNR of the link between source and the $i^{\text {th }}$ relay. Note that (6.14) represents the mutual information when the source-relay channel is used all the time. However, in the DF protocol, the relay sourcerelay link is only used for $T_{1}$ out of every $T$ symbol periods. Thus, the mutual information needs to be scaled by the ratio $T_{1} / T$ when computing the probability that a relay is in an outage.

The information-outage probability of the link from the source to node $N_{i}$ is

$$
p_{i}=\operatorname{Pr}\left[\frac{T_{1}}{T} I\left(\mathbf{s}, \mathbf{r}_{i} \mid f_{i}\right)<r\right]
$$

where $r$ is the rate of the error control code. Substituting (6.14) into (6.15) gives

$$
\begin{aligned}
p_{i} & =\operatorname{Pr}\left[\frac{T_{1}}{T} \log _{2}\left(1+P_{1}\left|f_{i}\right|^{2}\right)<r\right] \\
& =\operatorname{Pr}\left[\left|f_{i}\right|^{2}<\Gamma_{1}\right]
\end{aligned}
$$

where

$$
\Gamma_{1}=\frac{2^{r T / T_{1}}-1}{P_{1}} .
$$

Equation (6.16) is the cumulative distribution function (CDF) of the random variable $\left|f_{i}\right|^{2}$ evaluated at $\Gamma_{1}$. If we assume that $f_{i}$ is circularly-symmetric complex Gaussian with zero mean and unit variance, then $\left|f_{i}\right|^{2}$ will be exponential with unit mean. By recalling the $\mathrm{CDF}$ of an exponential random variable, the information-outage probability is

$$
p_{i}=1-e^{-\Gamma_{1}} .
$$

Because the $f_{i}$ 's are independent and identically distributed (i.i.d.) and the threshold $\Gamma_{1}$ is common to all relays, $p_{i}$ is the same at all $R$ relays and may be denoted as $p$.

Let $Z_{i}=\{0,1\}$ be an indicator variable that equals unity when the $i^{\text {th }}$ relay is in an outage. $Z_{i}$ is a Bernoulli random variable with $P\left[Z_{i}=1\right]=p$. The number 
of relays $K=|\mathcal{K}|$ that successfully decode the first-phase transmission is $K=$ $\sum_{i=1}^{R} Z_{i}$. Because the channels are independent, so are the $Z_{i}$ 's, and it follows that $K$ is a binomial random variable. The probability that the random variable $K$ is equal to $k$ is given by the probability mass function (pmf) of $K$,

$$
\begin{aligned}
p_{K}[k] & =\operatorname{Pr}[K=k] \\
& =\left(\begin{array}{l}
R \\
k
\end{array}\right)(1-p)^{k} p^{R-k} .
\end{aligned}
$$

The second-phase transmission may also be characterized in terms of an outage probability. However, the outage probability at the destination depends on the number of nodes $K$ in the decoding set as well as the maximum number of nodes $K_{\max }$ that may transmit during the second phase of the protocol. Define the conditional end-to-end information-outage probability for the second phase of the DF protocol as

$$
\operatorname{Pr}[\text { Outage } \mid k]=\operatorname{Pr}[I(S, \mathbf{x} \mid \mathbf{h})<r \mid K=k]
$$

where $S$ is the space-time codeword and $\mathbf{h}$ is a length $\min \left(k, K_{\max }\right)$ vector containing the coefficients $g_{i}$ corresponding to those relays that transmit during the second phases. Equation (6.20) represents the probability that the destination is in an outage during the second phase given that the decoding set has $k$ relays in it.

When using a rate $T_{1} / T_{2}$ orthogonal STBC over a point-to-point link, the mutual information is [11]

$$
I(S, \mathbf{x} \mid \mathbf{h})=\frac{T_{1}}{T_{2}} \log _{2}\left(1+P_{2}\|\mathbf{h}\|^{2}\right) .
$$

This mutual information expression assumes full use of the channel. However, in the DF protocol, the relay-destination link is only active for $T_{2}$ out of every $T$ channel uses, and thus (6.21) must be scaled by $T_{2} / T$.

Substituting the scaled version of (6.21) into (6.20) results in

$$
\begin{aligned}
\operatorname{Pr}[\text { Outage } \mid k] & =\operatorname{Pr}\left[\frac{T_{1}}{T} \log _{2}\left(1+P_{2}\|\mathbf{h}\|^{2}\right)<r \mid K=k\right] \\
& =\operatorname{Pr}\left[\|\mathbf{h}\|^{2}<\Gamma_{2} \mid K=k\right]
\end{aligned}
$$

where

$$
\Gamma_{2}=\frac{2^{r T / T_{1}}-1}{P_{2}} .
$$

This is the CDF of $\|\mathbf{h}\|^{2}$ evaluated at $\Gamma_{2}$. When there are $\min \left(k, K_{\max }\right)$ relays transmitting in the second phase, then $\|\mathbf{h}\|^{2}$ is Erlang-m with $\min \left(k, K_{\max }\right)$ degrees of freedom. Using the CDF of an Erlang-m distribution, the outage probability becomes

$$
\operatorname{Pr}[\text { Outage } \mid k]=1-\sum_{n=0}^{\min \left(k, K_{\max }\right)-1} \frac{\Gamma_{2}^{n}}{n !} e^{-\Gamma_{2}} .
$$


Of course, when $k=0, \operatorname{Pr}[$ Outage $\mid k]=1$ since the system is always in an endto-end outage when no relays receive the source transmission (recall that we assume no direct link from source to destination).

From the theorem on total probability, the overall end-to-end outage probability may be found from the conditional outage probabilities as

$$
\operatorname{Pr}[\text { Outage }]=\sum_{k=0}^{R} p_{K}[k] \operatorname{Pr}[\text { Outage } \mid k] .
$$

By substituting (6.19) and (6.24) into (6.25), we get the following expression for end-to-end outage probability

$$
p_{D}=p^{R}+\sum_{k=1}^{R}\left(\begin{array}{l}
R \\
k
\end{array}\right)(1-p)^{k} p^{R-k}\left(1-\sum_{n=0}^{\min \left\{k, K_{\max }\right\}-1} \frac{\Gamma_{2}^{n}}{n !} e^{-\Gamma_{2}}\right) .
$$

\subsubsection{Numerical results}

By using (6.26), we can determine the outage probability for a network comprised of $R$ relays that uses a particular space-time code. Consider two examples, one that uses the transposed Alamouti code with dispersion matrices given by (6.4), and another that uses the orthogonal STBC with dispersion matrices given by (6.12). Both systems use a rate $r=1 / 2$ error control code. With the Alamouticoded system, no more than $K_{\max }=2$ relays may transmit during the second phase, while with the other orthogonal system, no more than $K_{\max }=4$ relays may transmit. Let $K^{\prime}=\min \left(K, K_{\max }\right)$ be the number of relays that actually transmit during the second phase, where $K$ is the number of relays that successfully decoded the source's transmission. The total transmitted power of all relays is $P=P_{1}+K^{\prime} P_{2}$. As explained later in this section, the powers $P_{1}$ and $P_{2}$ are selected to minimize the outage probability subject to the total power constraint.

Figure 6.3 shows the information-outage probability as a function of SNR for both space-time codes and a variable number of relays. Since the noise power is unity, the SNR is equal to the total power $P$. For the Alamouti-coded system, the number of relays is between 2 and 8 , while for the other orthogonal system, the number of relays is between 4 and 10. For both systems, performance improves with increasing $R$. Even though no more than $K_{\max }$ relays can be used during the second transmission phase, it is still advantageous to have more than $K_{\max }$ relays present in the network. This is due to the diversity present in the first phase transmission. Having more than $K_{\max }$ relays makes it more likely that at least $K_{\max }$ relays will be able to decode the source's transmission, and thus it is very likely that $K_{\max }$ relays will transmit the entire space-time codeword during the second phase. From the curves, it is seen that the Alamouti code provides a diversity order of $K_{\max }=2$ while the other orthogonal system provides a 


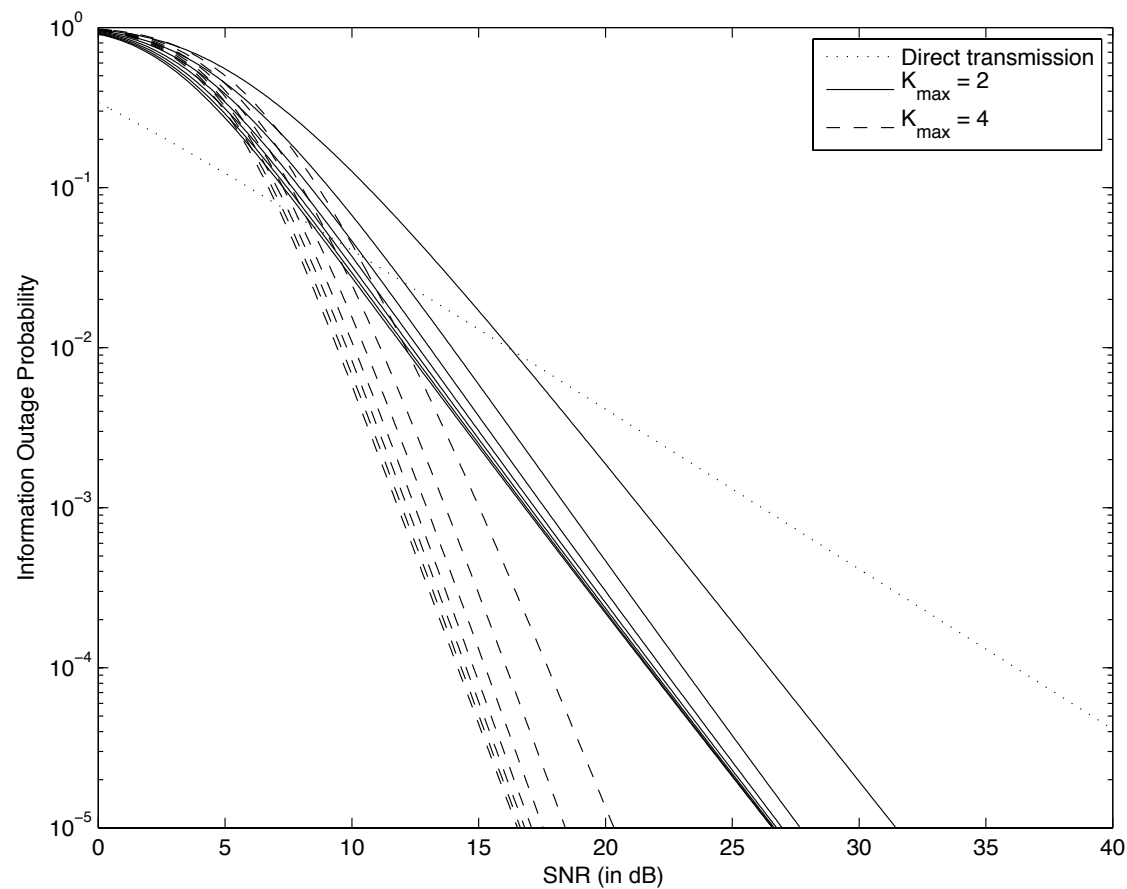

Figure 6.3: Comparison of the information-outage probability of several systems: Direct, DF using the Alamouti code and up to $K_{\max }=2$ transmitting relays, DF using orthogonal STBC and up to $K_{\max }=4$ transmitting relays. For each value of $K_{\max }$, a set of seven curves is shown corresponding to a different number of relays $R$. For the $K_{\max }=2$ system, the total number of relays $R$ is between 2 and 8, while for the $K_{\max }=4$ system there were between 4 and 10 relays. For each value of $K_{\max }$ performance improves with increasing $R$.

diversity order of $K_{\max }=4$. Using additional relays does not improve the overall system's diversity order, but it does provide an additional coding gain.

Also shown in Figure 6.3 is the performance of a direct point-to-point link transmitting with transmission power $P=$ SNR and using a single antenna at each end of the communication link. Because this is just a single-input singleoutput (SISO) system, the diversity order is only equal to one, and thus asymptotically the performance of the direct transmission is worse than the considered DF protocols. However, at very low SNR, the performance of the direct link is actually better than the systems that use a distributed STBC. This is because the system using a direct link may concentrate all of its power into the single transmission rather than diluting the energy across the transmissions of the two hops. Thus, at very low SNR, a direct link might be more effective than using the distributed STBC. However, keep in mind that these results assume that all channels have unity power gain. When relays are placed between the source and destination, then it is possible that channels used by the system with the 

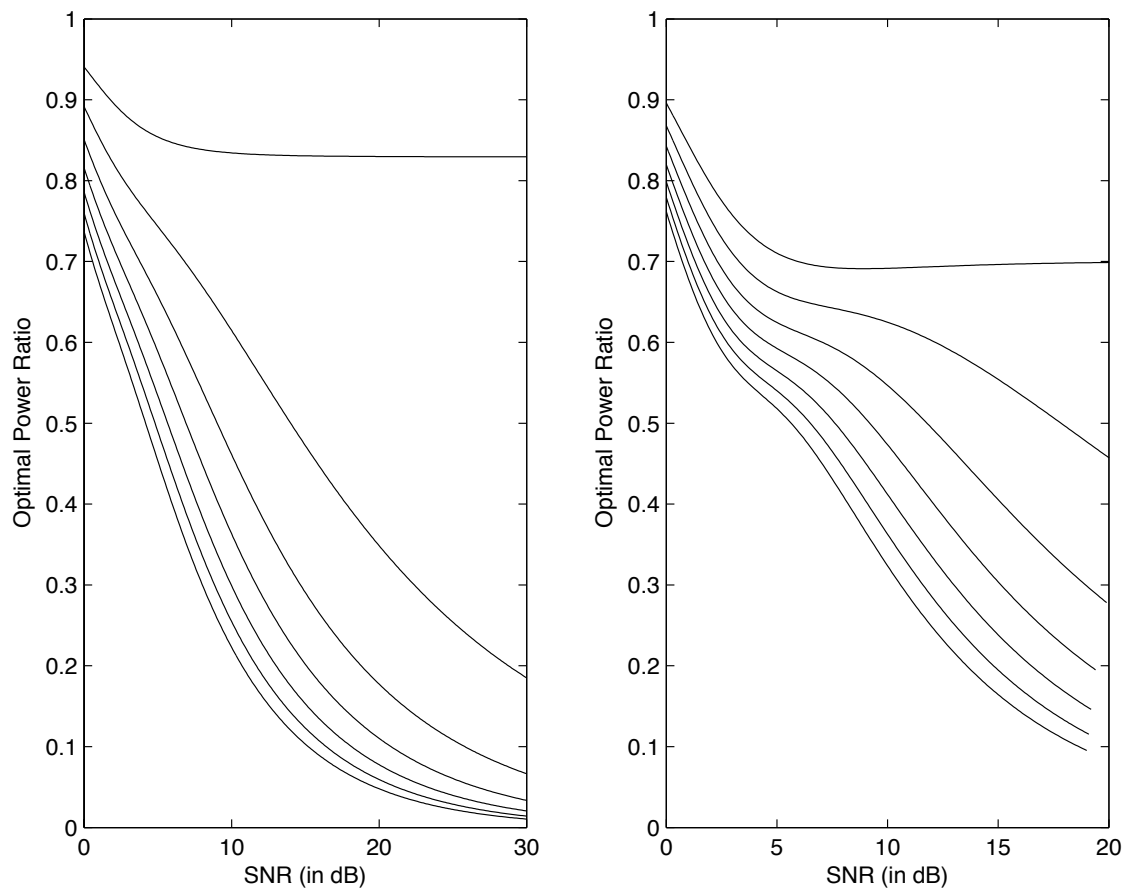

Figure 6.4: The optimal power ratio for the $K_{\max }=2$ system and between 2 and 8 relays (left subfigure) and the $K_{\max }=4$ system with between 4 and 10 relays (right subfigure). The optimal power ratio decreases with increasing $R$.

distributed STBC (i.e., the gains from source to relays and the gains from relays to destination) will have a higher gain than the channel used by the system that uses direct transmission.

Let $P_{1} /\left(K^{\prime} P_{2}\right)$ be the ratio of the power used during the first phase to the power used during the second phase. The results shown in Figure 6.3 assume that the power is selected to minimize the information-outage probability subject to the total power constraint $P=P_{1}+K^{\prime} P_{2}$. To find the optimal power ratio for each SNR point, we compute the information-outage probability for all ratios between $10^{-4}$ and 1 in increments of $10^{-4}$, and pick the ratio that minimizes the information-outage probability. The result of this optimization is shown in Figure 6.4. The left subfigure shows the optimal power ratios for the $K_{\max }=2$ system (Alamouti coded) and the right subfigure shows the optimal power ratios for the $K_{\max }=4$ system. For each system, a family of curves is shown corresponding to the different values of $R$ considered in Figure 6.3. The power ratio decreases with increasing $R$ and with increasing SNR. This is because as $R$ grows, the likelihood that $K_{\max }$ relays can decode the source's signal improves as a function of $P_{1}$, and thus the system can afford to decrease $P_{1}$ and devote more power to the second phase transmission. 


\subsection{Amplify-and-forward distributed STBC}

In contrast to decode-and-forward protocols, amplify-and-forward protocols do not require that each relay fully demodulates and decodes the signal it receives from the source. Instead, relay $N_{i}$ obtains the vector $\mathbf{r}_{i}$ given in (6.1) by downconverting the received signal to baseband and passing it through a pair of filters matched to the in-phase and quadrature basis functions. The matched-filters are sampled at the symbol rate, resulting in a set of $T_{1}$ complex samples that are placed into the vector $\mathbf{r}_{i}$. Rather than demodulating and decoding $\mathbf{r}_{i}$, the relay transmits a linear combination of the samples in $\mathbf{r}_{i}$ and its conjugates at power $P_{2}$ [8].

We can write the normalized signal transmitted by node $N_{i}$ in vector form as

$$
\mathbf{t}_{i}=\sqrt{\frac{1}{P_{1}+1}}\left(A_{i} \mathbf{r}_{i}+B_{i} \overline{\mathbf{r}}_{i}\right)
$$

where $A_{i}$ and $B_{i}$ are the dispersion matrices assigned to node $N_{i}$. The two major differences between (6.27) and the DF transmitted signal in (6.3) are 1) in AF, the transmitted signal is a linear combination of the samples in the received vector $\mathbf{r}_{i}$ (and its conjugates) rather than a linear combination of the re-modulated symbols in the vector $\mathbf{s}$ (and its conjugates), and 2) because the noise power is unity, the average received signal power is $P_{1}+1$, and the scaling $\sqrt{\frac{1}{P_{1}+1}}$ is required to normalize the signal.

For the important special case where either $A_{i}$ or $B_{i}$ is zero, we can simplify the transmitted signal similar to $(6.7)$ as

$$
\mathbf{t}_{i}=\sqrt{\frac{1}{P_{1}+1}} C_{i} \mathbf{r}^{(i)}
$$

where

$$
\mathbf{r}^{(i)}= \begin{cases}\mathbf{r}, & \text { if } B_{i}=0 \\ \overline{\mathbf{r}}, & \text { if } A_{i}=0\end{cases}
$$

and $C_{i}$ is as given by $(6.5)$.

Using (6.2) and assuming perfect symbol-level synchronization, the resulting received signal vector at the destination can be written as

$$
\mathbf{x}=\sum_{i=1}^{R} g_{i} \sqrt{P_{2}} \mathbf{t}_{i}+\mathbf{w} .
$$

Substituting (6.1) into (6.29) and (6.30) results in

$$
\mathbf{x}=\sum_{i=1}^{R} g_{i} f_{i} \sqrt{\frac{P_{1} P_{2}}{P_{1}+1}} C_{i} \mathbf{s}^{(i)}+\underbrace{\sqrt{\frac{P_{2}}{P_{1}+1} \sum_{i=1}^{R} g_{i} C_{i} \mathbf{v}_{i}+\mathbf{w}} .}_{\boldsymbol{n}}
$$


This may compactly be represented as

$$
\mathbf{x}=\sqrt{\frac{P_{1} P_{2}}{P_{1}+1}} S \mathbf{h}+\boldsymbol{n}
$$

where the $T_{2} \times R$ space-time codeword $S$ is as given in (6.9), the channel vector is

$$
\mathbf{h}=\left[\begin{array}{c}
f_{1} g_{1} \\
f_{2} g_{2} \\
\vdots \\
f_{R} g_{R}
\end{array}\right]
$$

and the complex noise vector $\boldsymbol{n}$ is Gaussian when conditioned on the $\left\{g_{i}\right\}$ and will generally be colored because the signal transmitted by the relay will contain a linear combination of the elements of the white noise vector $\mathbf{v}_{i}$.

Compared with the DF case (6.8), the AF received signal in (6.32) differs in 3 ways: 1) all $R$ relays transmit a signal during the second phase, not just those that can decode the source's transmission, 2) the channel vector consists of the product of the source-relay and relay-destination channel gains instead of just the relay-destination channel gains, and 3) the additive noise will have a higher power and will generally be colored.

As was the case for DF, the $T_{2} \times R$ matrix $S$ in (6.32) plays the same role as a space-time code matrix in a conventional point-to-point multiple-input multipleoutput (MIMO) system, except that in the distributed space-time code scenario, the matrix is generated without access to s. For this reason, we say that $S$ defines a distributed space-time code operating in AF mode. We can think of $\mathbf{h}$ as the equivalent channel matrix and $\boldsymbol{n}$ as additive noise, although $\boldsymbol{n}$ is clearly a function of the space-time code. Because of the similarities to conventional STBCs, we can analyze the diversity gain and coding gain performance of this family of distributed AF space-time codes using the the same technique we use for conventional codes, i.e., bounding the pairwise error probability.

\subsubsection{Performance analysis}

The achievable diversity of linear dispersion (LD) codes operating in an AF system can be determined using the same technique that is often used for point-topoint space-time coded systems, i.e., by bounding the pairwise error probability. The exact results are complex and we refer to the reader to [8] for details. The main result, however, is that the achievable diversity is

$$
d=R\left(1-\frac{\log \log P}{P}\right)
$$

which is achieved whenever the $S_{k}-S_{l}$ is full rank for all $l \neq k$. This result converges to $R$ for very large power $P$, so LD coding operating in AF systems 
achieves approximately the same diversity as a point-to-point system with $R$ antennas $^{2}$.

Interestingly, when the number of relays is large and the power is also large, the coding gain for distributed LD codes is the same as for LD codes operating in point-to-point systems. On the other hand, when the power $P$ is moderate, the code matrices should be designed such that the code is "scale-free", i.e., it should perform well when some relays are not working. Mathematically, this requires the codeword difference matrices to remain full rank when some columns are deleted.

\subsubsection{Practical distributed STBC for AF systems}

Although arbitrary LD codes can achieve almost full diversity with mild conditions on the $A_{i}$ matrices, they are generally difficult to decode because maximum likelihood decoding, i.e.,

$$
\arg \min _{\mathbf{s}}\left\|\mathbf{x}-\sqrt{\frac{P_{1} P_{2}}{P_{1}+1}} S \mathbf{h}\right\|
$$

has high computational complexity for the general case. This problem can be alleviated by using extending well-known orthogonal [15] or quasi-orthogonal [7] code designs for point-to-point systems to the distributed scenario, as discussed in [9]. The results are distributed codes that are fully diverse, allow low-complexity decoding, and are scale-free, yielding good coding gain for moderate transmit powers. Because the noise vector $\boldsymbol{n}$ is not generally white, true-ML detction cannot be achieved through linear processing methods such as the decoupleddecoding approach commonly used for orthogonal codes operating over pointto-point links. However, as was reported in [9], the performance when using decoupled decoding is only slightly inferior to that of using true-ML detection (i.e. around $0.5 \mathrm{~dB}$ ).

\subsection{The synchronization problem}

One of the key challenges to designing high-performance distributed space-time coded systems is symbol-level synchronization among the relay nodes. In conventional point-point space-time coded MIMO systems, co-located antennas obviate this issue. In cooperative systems, sometimes described as virtual MIMO, the antennas are separated by wireless links. One approach is simply to use appropriate hardware and higher-layer protocols to ensure that transmissions from every participating relay are synchronized. Unfortunately, this may not be pos-

2 Note that here we assume there that there is no information passed directly between the source and destination. If such a link exists, the diversity result simply increases by 1 . 
sible in practice and, in any case, it would require significant signaling overhead that may dramatically increase bandwidth requirements. Other approaches that effectively circumvent the synchronization problem include delay diversity, delay tolerant distributed space-time codes, and space-time spreading. We will next consider each of these approaches briefly.

\subsubsection{Delay diversity}

It is well known that point-point communication over multipath fading channels provides diversity that can be exploited by appropriate receiver design [18]. In cases where Intersymbol Interference (ISI) is negligible, as is common in spreadspectrum systems, RAKE reception is sufficient. When ISI cannot be ignored, maximum likelihood sequence detection can be performed using the Viterbi algorithm to extract full diversity in the number of resolvable paths. Mathematically, this involves transforming the frequency selective SISO system into an equivalent flat-fading multi-input single-output (MISO) system that uses a particular spacetime code induced by the frequency selective channel. Interestingly, the reverse is also possible. That is, we can transform a flat fading MISO system into a virtual frequency selective SISO system by using a space-time code described by the following scheme: in the first time slot, the symbol $x[1]$ is transmitted on antenna 1 and all other antennas are silent. In the second time slot, $x[1]$ is transmitted from antenna 2 and $x[2]$ is transmitted by antenna 1 and all other antennas remain silent. At time slot $m, x[m-l]$ is transmitted on antenna $l+1$ for $l=0,1, \ldots L-1$. This transmission scheme yields a received signal that is identical to that received in a SISO frequency selective channel with $L$ paths. This special point-point space-time coding scheme is called delay diversity [22].

Delay diversity cannot be implemented in cooperative communication systems in exactly this way without requiring what we are trying to avoid, i.e., synchronization to determine which relay transmits which symbol in which order. Fortunately, it is straightforward to implement delay diversity in a distributed manner. The simplest way to do this is simply for the relays to wait a random amount of time before they re-transmit the symbol or signal they have most recently received. The destination will receive a signal that is equivalent to that received in a SISO multipath channel, so full diversity will be achievable (with probability 1), assuming maximum likelihood detection at the destination. Linear detectors/equalizers can also be used at the destination, e.g., minimum meansquared error (MMSE), or decorrelating equalization, with some diversity loss. Interestingly, MMSE detection in conjunction with serial interference cancellation (decision feedback implementation) achieves full diversity [21] with much lower complexity than ML detection when the number of relays is large. 


\subsubsection{Delay tolerant space-time codes}

Another approach to distributed space-time coding without synchronization involves the use of so-called delay tolerant distributed space-time codes whose performance is insensitive to delays among the received signals from each relay.

It is well known that the diversity order of a space-time block code is equal the minimum rank of the difference matrix over all pairs of distinct code matrices [15]. A space-time code is said to be $\tau$-delay tolerant if for all distinct code matrices $S_{k}$ and $S_{\ell}$, the difference matrix $S_{k}-S_{\ell}$ retains full rank even though the columns of the code matrices are transmitted or received with arbitrary delays of duration at most $\tau$ symbols. Let $S$ be a codeword matrix from a synchronized space-time block code, as in (6.9), and let $\Delta S$ be the code matrix received at the destination due to transmission or propagation delays. Then $\Delta S$ can be written as

$$
\Delta S=\left[\begin{array}{cccc}
0^{\Delta_{1}} & 0^{\Delta_{2}} & \cdots & 0^{\Delta_{R}} \\
C_{1} \mathbf{s}^{(1)} & C_{2} \mathbf{s}^{(2)} & \cdots & C_{R} \mathbf{s}^{(R)} \\
0^{\tau-\Delta_{1}} & 0^{\tau-\Delta_{2}} & \cdots & 0^{\tau-\Delta_{R}}
\end{array}\right] .
$$

The collection of all such codewords $\Delta S$ constitutes a $\tau$-delay tolerant space-time code if for all delay profiles $\left\{\Delta_{k}\right\}_{k=1}^{R}$ such that $\Delta_{k} \leq \tau$ for all $k$, it achieves the same diversity as the synchronized code. Work on delay-tolerant codes under this framework includes $[13,5,3,16]$. Although delay diversity extracts full diversity in the number of relays, delay tolerant space-time codes promise better coding gain and, in some circumstances, lower decoding complexity.

\subsubsection{Space-time spreading}

Delay diversity is successful in achieving full diversity in part because the distinct delays for the received signals from each relay provide a unique signature enabling the receiver to separate each resolvable path before co-phasing and combining. A similar unique signature can be implemented with coding, i.e., space-time spreading (STS).

One of the simplest space-time spreading strategies is to assign the source and each relay a unique spreading code, as in code-division multiple access (CDMA) communications. When the relays are not synchronized, the signal received at the destination will be similar to that obtained in a conventional (non-cooperative) asynchronous CDMA uplink, so that the transmissions from the source and each relay can be separated using well-known multiuser detection (MUD) signal processing strategies, co-phased, and re-combined to extract full diversity without symbol-level synchronization. Note that, although CDMA is a spread-spectrum signaling format, it does not need to operate in a spectrally inefficient mode. In fact, it was shown in [19] that the information outage probability of an asynchronous cooperative CDMA uplink under decorrelating multiuser detection is minimized when the system is slightly overloaded, i.e., when the number of relays 
is slightly larger than the processing gain. This is not surprising because an overloaded CDMA system is operating at high spectral efficiency.

A more sophisticated space-time spreading strategy was designed in [14], which does not require synchronization among the relays, channel estimation, or complex multiuser signal processing at the destination or relays. The necessity of channel information is obviated by the use of differentially-encoded symbols from each source, as in [4], during the first transmission phase. During the second transmission phase, dedicated relays use a STS AF strategy described in [4] that allows for low complexity decoding and large diversity gain without channel estimation. Because high-complexity multiuser detection strategies are not used here, the residual multiple access interference (MAI) and ISI must be mitigated by the use of specially designed spreading codes that provide an "interference free window" (IFW) where the off-peak aperiodic autocorrelation and crosscorrelation values become zero, resulting in zero MAI and ISI, provided the maximum asynchronous delay is within the IFW [20]. The resulting system extracts full diversity without channel knowledge or complex multiuser detection at the destination or relays.

\subsection{Conclusion}

Distributed space-time block codes are able to effectively exploit the spatial diversity present in a multi-relay network. With a distributed-space time code, each relay transmits a particular column of a space-time codeword. The decodeand-forward strategy is appropriate when there are more relays than there are columns in the space-time codeword, since only a subset of relays may participate in the transmission of the distributed space-time codeword, namely those that receive the source's transmission. However, DF protocols require coordination among the relays to assure that each relay transmits a specific column of the space-time codeword. Amplify-and-forward protocols are well suited to the case that the number of relays is equal to the number of columns in the space-time code, since with AF protocols every relay participates in the transmission of the space-time codeword regardless of the quality of the source-relay transmission.

In addition to the implementation challenges that are common to conventional MIMO systems, the lack of synchronization at the destination receiver imposes additional challenges to systems that use distributed space-time codes. The synchronization problem can be alleviated by using delay diversity, spacetime spreading, or delay-tolerant space-time codes. 


\section{References}

[1] S. M. Alamouti, "A simple transmit diversity technique for wireless communications," IEEE Journal on Selected Areas in Communications, 16, 1998, 1451-1458.

[2] M. O. Damen, A. Chkeif, and J. Belfiore, "Lattice code decoder for space-time codes," IEEE Communications Letters, 4, 2000, 161-163.

[3] M. O. Damen and A.R. Hammons, "Delay-tolerant distributed TAST codes for cooperative diversity," IEEE Transactions on Information Theory, 53, 2007, 3755-3773.

[4] M. El-Hajjar, O. Alamri, S. X. Ng, and L. Hanzo, "Turbo detection of precoded sphere packing modulation using four transmit antennas for differential space-time spreading," IEEE Transactions on Wireless Communications, 7, 2006, 943-952.

[5] A. R. Hammons and M. O. Damen, "On delay-tolerant distributed space-time codes," in Proc. of IEEE Military Communications Conference (MILCOM), 2007.

[6] B. Hassibi and B. M. Hochwald, "High-rate codes that are linear in space and time," IEEE Transactions on Information Theory, 48, 2002, 1804-1824.

[7] H. Jafarkhani, "A quasi-orthogonal space-time block code," IEEE Transactions on Communications, 49, 2001, 1-4.

[8] Y. Jing and B. Hassibi, "Distributed space-time coding in wireless relay networks," IEEE Transactions on Wireless Communications, 5, 2006, 3524-3536.

[9] Y. Jing and H. Jafarkhani, "Orthogonal and quasi-orthogonal designs in wireless relay networks," IEEE Transactions on Information Theory, 53, 2007, 4106-4118.

[10] J. N. Laneman and G. W. Woernell, "Distributed space-time-coded protocols for exploiting cooperative diversity in wireless networks," IEEE Transactions on Information Theory, 49, 2003, 2415-2425.

[11] E. G. Larsson and P. Stoica, Space-time block Coding for Wireless Communications. Cambridge University Press, 2008.

[12] R. Nabar, H. Bolcskei, and F. Kneubuhler, "Fading relay channels: Performance limits and space-time signal design," IEEE Journal on Selected Areas in Communications, 22, 2004, 1099-1109.

[13] Y. Shang and X. G. Xia, "Shift-full-rank matrices and applications in spacetime trellis codes for relay networks with asynchronous cooperative diversity," 
IEEE Transactions on Information Theory, 52, 2006, 3153-3167.

[14] S. Sugiura, S. Chen, and L. Hanzo, "Cooperative differential space-time spreading for the asynchronous relay aided CDMA uplink using interference rejection spreading code," IEEE Signal Processing Letters, 17, 2010, 117-120.

[15] V. Tarokh, H. Jafarkhani, and A. Calderbank, "Space-time block codes from orthogonal designs," IEEE Transactions on Information Theory, 45, 1999, 1456-1467.

[16] M. Torbatian and M. O. Damen, "On the design of delay-tolerant distributed space-time codes with minimum length," IEEE Transactions on Wireless Communications, 8, 2009, 931-939.

[17] D. Torrieri and M. C. Valenti, "Efficiently decoded full-rate space-time block codes," IEEE Transactions on Communications, 58, 2010, 480-488.

[18] D. Tse and P. Viswanath, Fundamentals of Wireless Communication. Cambridge University Press, 2005.

[19] K. Vardhe, D. Reynolds, and M.C. Valenti, "The performance of multiuser cooperative diversity in an asynchronous CDMA uplink," IEEE Transactions on Wireless Communications, 7, 2008, 1930-1940.

[20] H. Wei, L. Yang, and L. Hanzo, "Interference-free broadband single and multicarrier DS-CDMA," IEEE Communications Magazine., 43, 2005, 68-73.

[21] S. Wei, D. L. Goeckel, and M. C. Valenti, "Asynchronous cooperative diversity," IEEE Transactions on Wireless Communications, 5, 2006, 1547-1557.

[22] A. Wittneben, "A new bandwidth efficient transmit antenna modulation diversity scheme for linear digital modulation," in Proc. of IEEE International Conference on Communications (ICC), 1993. 